
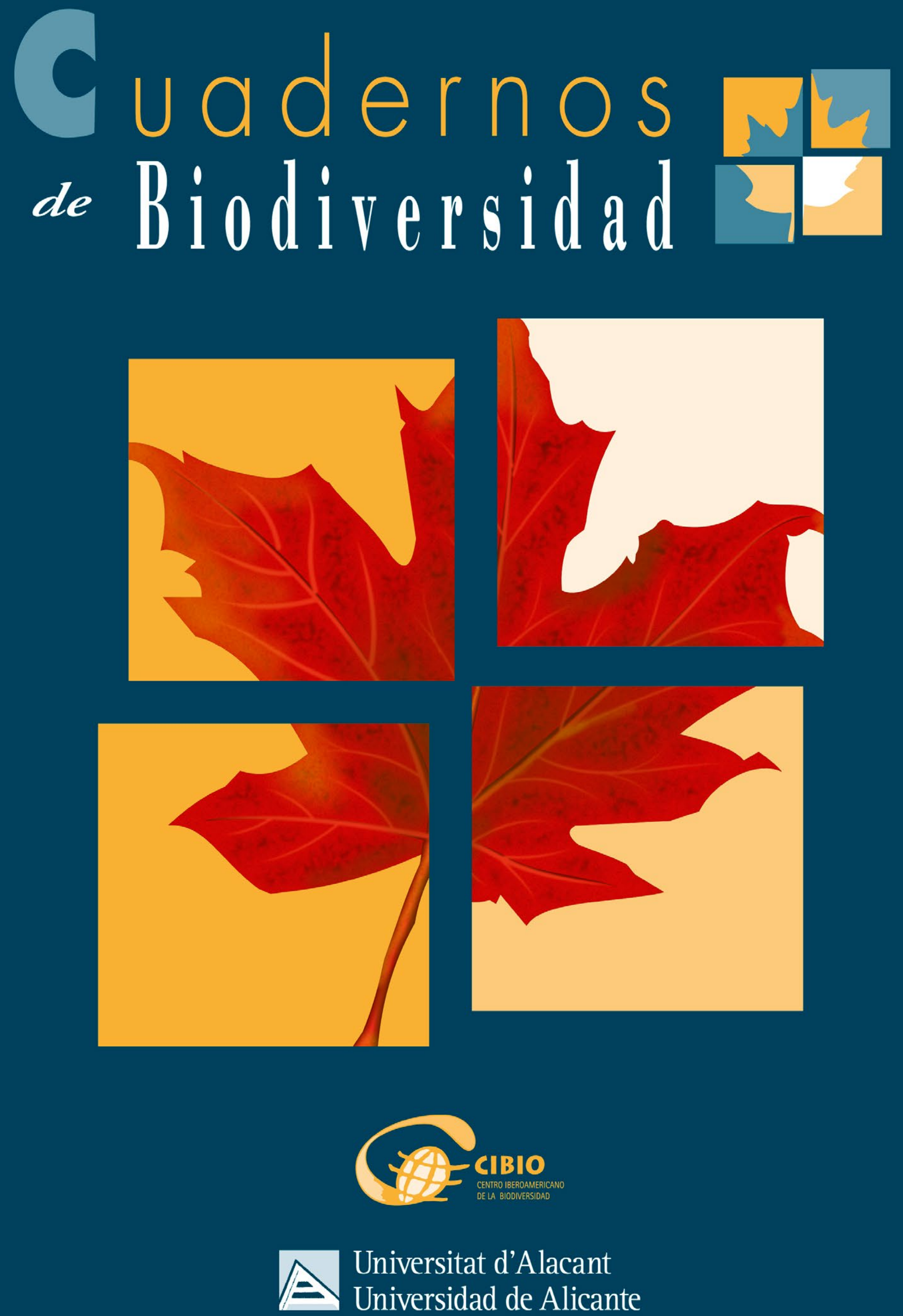


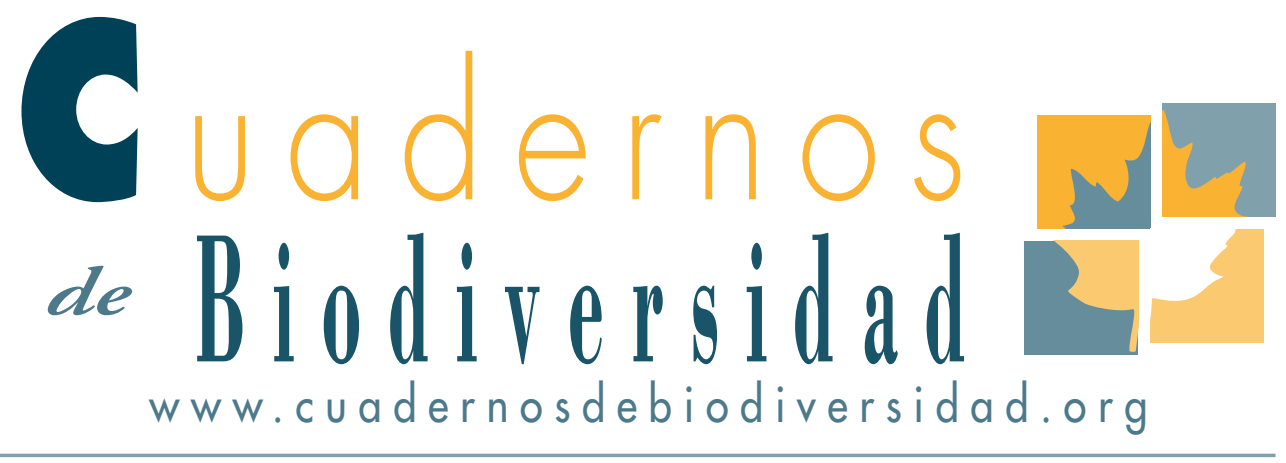

\section{Colección ex situ de plantas vivas de especies nativas del orden Zingiberales del Sur del Lago de Maracaibo,} Venezuela

\section{S. Rangel Marquina ${ }^{I^{*}}$, J. P. Uzcátegui Varela ${ }^{1}, K$. Dávila Zambrano ${ }^{2}$, A. Briceño Rangel', M. Ceballos Ramirez ${ }^{1}$, J. A. Mercado Marquez ${ }^{1}$}

* RANGELS@UNESUR.EDU.VE

i Grupo de Investigaciones en Ciencias Animales y Plantas Tropicales. Universidad Nacional Experimental Sur del Lago “Jesús María Semprum”. Núcleo la Victoria estado Mérida, VENEZUELA

2 Grupo de Investigaciones en Agroecología. Universidad Nacional Experimental Sur del Lago "Jesús María Semprum". Núcleo la Victoria estado Mérida, Venezuela

\section{ABSTRACT}

The loss of biological diversity in the Southern Part of the Lago de Maracaibo, Venezuela, due to cattle rising, has caused the extinction of $97 \%$ of the original the Zingiberales order for purpose of study and conservation, because of the importance of this subject in the tropical floriculture (cut flowers, foliage and landscaping). The use of the biological diversity and the new native species flora could promote the efficient and sustainable use of the natural resources of the Southern Part of the Lago de Maracaibo. For that reason, it was collected 18 samples among varieties and species which passed the nursery stage, and then they were transplanted in the field after three months, besides, it was accounted the humidity and light conditions they had in their natural ecosystem; as a result plants showed favorable adaptability indicators.

Key words: Biological diversity, ex situ conservation, Heliconiaceae, rainforest relict, Zingiberales. 


\section{INTRODUCCIÓN}

El Sur del lago de Maracaibo es una eco-región que comprendía un tipo de selva tropical húmeda que fue transformada y sustituida por un sistema de reemplazo ganadero, en donde los grandes árboles y exuberante vegetación fueron sustituidos por pastizales en un proceso que comenzó en 1940 y para la fecha actual queda solo un 3\% restringido a pequeños relictos selváticos, amenazados por sus propietarios ante el temor que sean declaradas tierras ociosas (Rangel et al., 2014; Romero, 1995).

El proceso de transformación del paisaje del Sur del Lago de Maracaibo ha ocasionado una gran pérdida de diversidad biológica difícil de cuantificar, debido en gran parte a los escasos trabajos científicos realizados. Esta situación nos llama a emprender actividades para la conservación de su biodiversidad en los pocos relictos selváticos que aun sobreviven.

La conservación de la biodiversidad comprende dos tipos: la conservación in situ y la ex situ. La conservación in situ hace referencia a ecosistemas completos cuyas especies silvestres son protegidas. La conservación ex situ busca mantener germoplasma fuera de sus ambientes originales para reducir la presión sobre las poblaciones silvestres (De Viana et al., 2011). Los programas de conservación ex situ complementan la conservación in situ almacenando a largo plazo germoplasma y permite mediante su estudio un mejor conocimiento de las características anatómicas, fisiológicas y bioquímicas del material almacenado, pudiendo también proporcionar propágulos para su utilización en programas de investigación, educativos y en la reintroducción (Iriondo, 2001).

Uno de los grupos vegetales representativos del ecosistema selvático en proceso de desaparición en el Sur del Lago de Maracaibo y que además forma parte importante en la cultura de su población, es el orden Zingiberales. Este orden abarca un grupo de monocotiledóneas tropicales que está constituido por ocho familias: Musaceae, Strelitziaceae,
Lowiaceae, Heliconiaceae, Zingiberaceae, Costaceae, Cannaceae y Marantaceae. Dos de ellas: Musaceae y Lowiaceae no son originarias del neotrópico, mientras que Heliconeaceae y Cannaceae son exclusivas del trópico americano (Kress, 1990), aunque existe un grupo conformado por ocho especies de Heliconiaceae que se encuentran en el Pacifico sur (Kress, 1985).

La popularidad de este orden va en aumento debido a que además de la belleza y llamativos colores de las brácteas de la mayoría de sus inflorescencias, hojas ornamentales y potencial paisajista, pueden ser fuente de otros tipos de recursos como tintes y fibras (Hoyos, 1999) esto significa que, a diferencia de otros grupos vegetales, los Zingiberales cuenten con un gran interés por parte de los pobladores del Sur del Lago de Maracaibo en la búsqueda de nuevos recursos a partir de especies nativas.

Con respecto a la importancia del orden Zingiberales en el Sur del Lago de Maracaibo podemos señalar que algunas especies de la familia Heliconiaceae como Heliconia bihai L. y Heliconia latispatha Benth., son utilizadas como flor de corte, al igual que ocurre con algunas Costaceae como Costus malortiea$n u s \mathrm{H}$. Wendl., mientras que las Marantaceae Thalia geniculata L. y Calathea luthea (Aubl.) E. Mey. Ex Schult., son apreciadas por su follaje. Esta última especie es utilizada para envolver las hallacas, plato típico de la cocina Venezolana.

Como el almacenamiento de germoplasma tiene lugar en forma de colección de plantas y en bancos de germoplasma (Iriondo, 2001), en nuestro caso específico se estableció una colección de plantas de especies nativas del Sur del Lago de Maracaibo del orden Zingiberales, debido en parte a su importancia dentro de la floricultura tropical y el paisajismo. Esta colección busca suministrar material para estudios anatómicos, fisiológicos, propagación y manejo agronómico, y a la vez ser fuente de propágulos, de tal forma que se reduzca la presión sobre las plantas silvestres y un mejor aprovechamiento de las especies nativas. 


\section{ÁREA DE ESTUDIO}

El Sur del Lago de Maracaibo, Venezuela comprende unas 795.000 ha que van desde el pie de monte de la Cordillera de Mérida hasta las Ciénagas del Catatumbo del Lago de Maracaibo (Fig 1). Temperaturas promedio de $29,9^{\circ} \mathrm{C}$, humedad relativa de $85 \%$ y precipitaciones de $2000 \mathrm{~mm}$ anuales (Rangel et al., 2014).

Para nuestro caso de estudio, consideramos que las especies nativas del Orden Zingiberales eran las que se encontraban asociadas a los relictos de selva, por lo que el muestreo consistió en visitar los relictos selváticos desde los $400 \mathrm{~m} \mathrm{~s}$. n. m. en la Cordillera de Mérida hasta el nivel del mar en el parque nacional Ciénagas de Juan Manuel del estado Zulia. En el campo se identificaron las especies pertenecientes al orden Zingiberales de forma visual y utilizaron las Claves sugeridas por Costa et al. (2011) y Kress (1997). Se tomaron tres rizomas por especie, se trasladaron a un vivero perteneciente a la Universidad Nacional Experimental Sur del Lago “Jesús María Semprum” (UNESUR) núcleo la Victoria en el estado Mérida y se sembraron en una mezcla (1:1) de tierra y arena, pasado tres meses se llevaron a campo tomando en consideración las condiciones ecológicas de exposición a la radiación y la disponibilidad de agua que presentan en sus condiciones naturales.

La colección se estableció en terrenos que contaban con grandes árboles que le brindaron en muchos casos la sombra requerida por algunas especies, dichas áreas forman parte de la Hacienda la Victoria, sede de la UNESUR, cercano a la población de Santa Cruz de Mora en el estado Mérida, a 535 m s. n. m. en un Bosque Húmedo Premontano donde se ha establecido principalmente el cultivo de café y la ganadería como sistemas productivos.

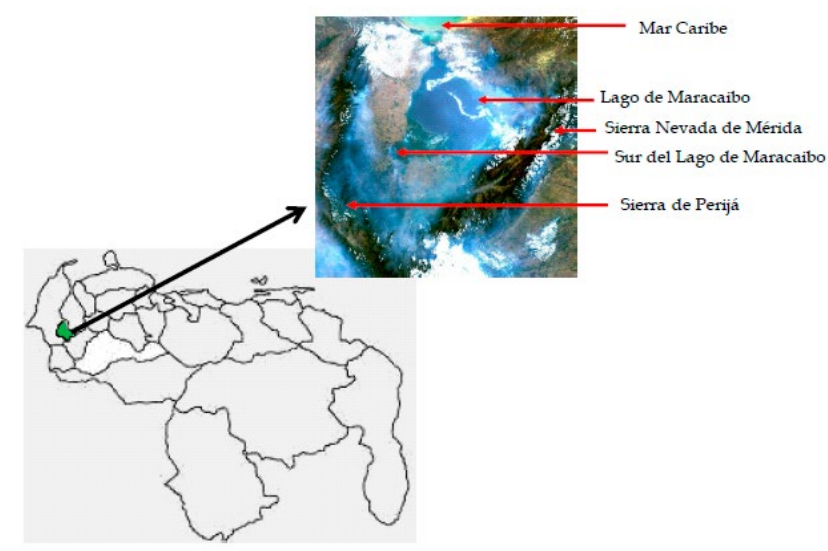

Figura 1. Área de Estudio. Sur del Lago de Maracaibo

\section{RESULTADOS}

Los rizomas recolectados se desarrollaron de manera satisfactoria en el sustrato en el que se plantó. A los 30 días se observó la emisión de hojas y a los 90 presentaron condiciones de tamaño y desarrollo suficiente como para ser llevados al lugar definitivo de plantación en el campo.

Se recolectó y se plantó en campo 16 especies de Zingiberales asociadas a los relictos selváticos que se distribuyen desde el pie de monte de la Sierra de Mérida hasta las Ciénagas de Juan Manuel en el Lago de Maracaibo.

El mayor número de especies recolectadas pertenecen a la familia Heliconiaceae (Tabla 1) entre ellas Heliconia bihai L. (Fig 2) presentó la más amplia distribución altitudinal seguida de Heliconia latispatha Benth. (Fig 3) debido a que se encontraron distribuidas desde el pie de monte en la Sierra de Mérida hasta las Ciénagas del Lago de Maracaibo. Contrariamente Heliconia marginata (Griggs) Pittier (Fig 4) se encontró casi exclusivamente en los suelos saturados de agua cerca del lago. 
Tabla 1. Especies de Zingiberales recolectadas en relictos selváticos distribuidos desde el pie de monte de la Sierra de Mérida hasta las Ciénagas de Juan Manuel en el Lago de Maracaibo y sus potenciales usos.

\begin{tabular}{|c|c|c|}
\hline Familia & Especie & Potenciales usos \\
\hline \multicolumn{3}{|c|}{ Heliconiaceae } \\
\hline & Heliconia bihai $\mathrm{L}$. & Flor de corte, paisajismo \\
\hline & Heliconia episcopalis Vell. & Flor de corte, paisajismo \\
\hline & Heliconia latispatha Benth. (Amarilla) & Flor de corte, paisajismo \\
\hline & Heliconia latispatha Benth. (Roja) & Flor de corte, paisajismo \\
\hline & Heliconia mariae Hook. f. & Flor de corte, paisajismo \\
\hline & Heliconia platystachys Baker & Flor de corte, paisajismo \\
\hline & Heliconia acuminata A. Rich. & Flor de corte, paisajismo \\
\hline & Heliconia hirsuta L. f. & Flor de corte, paisajismo \\
\hline & Heliconia marginata (Griggs) Pittier (Amarilla) & Flor de corte, paisajismo \\
\hline & Heliconia marginata (Griggs) Pittier (Roja) & Flor de corte, paisajismo \\
\hline \multicolumn{3}{|c|}{ Marantaceae } \\
\hline & Calathea lutea (Aubl.) E. Mey. Ex Schult. & Paisajismo, artesanía \\
\hline & Calathea inocephala (Kuntze) T. Durand \&B. D. jacks & Paisajismo \\
\hline & Thalia geniculata $\mathrm{L}$. & Follaje, paisajismo \\
\hline & Calathea latifolia (Will. ex Link) Klotzsch & Paisajismo \\
\hline \multicolumn{3}{|c|}{ Zingiberaceae } \\
\hline & Renealmia thyrsoidea (Ruiz \& Pav.) Poepp. \& Endl. & Paisajismo, tintes \\
\hline \multicolumn{3}{|l|}{ Costaceae } \\
\hline & Costus malortieanus $\mathrm{H}$. Wendl. & Paisajismo \\
\hline & Costus spiralis (Jacq.) Roscoe & Paisajismo, flor de corte \\
\hline \multicolumn{3}{|c|}{ Cannaceae } \\
\hline & Canna denudata Roscoe (Es un sinónimo de C. Paniculata Ruiz \&Pav.) & Paisajismo \\
\hline
\end{tabular}

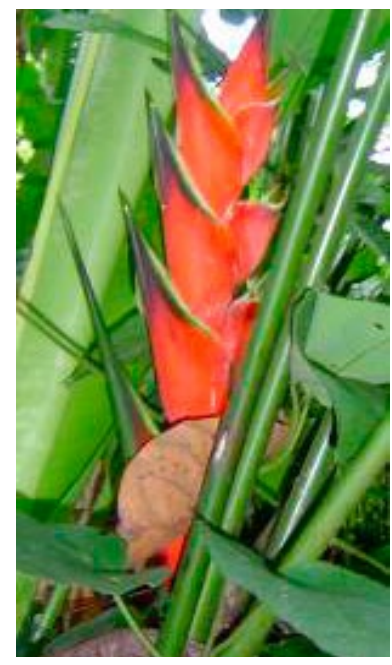

Figura 2. Heliconia bihai

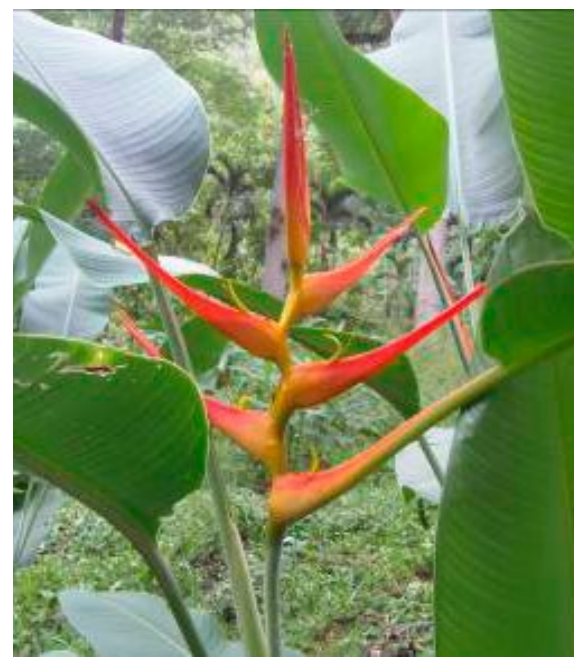

Figura 3. Heliconia latispatha

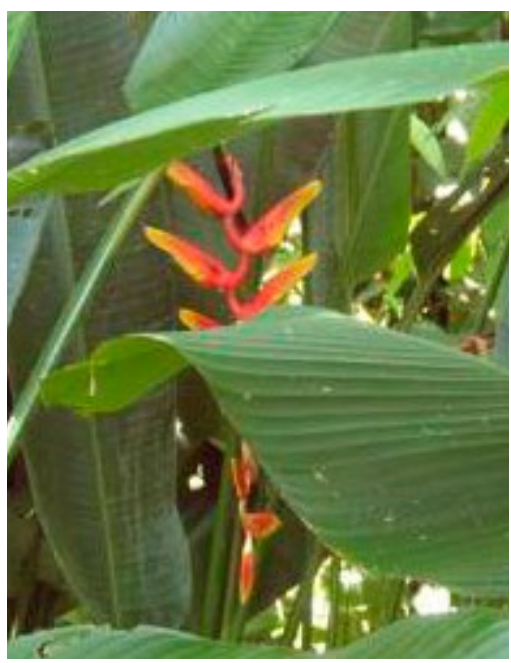

Figura 4. Heliconia marginata 
Por otra parte Heliconia mariae Hook. f., Helicona hirsuta L. f. y Heliconia platystachys Baker (Fig 5), se encontraron principalmente en los relictos selváticos ubicados en el pie de monte de la Sierra de Mérida, mientras que Heliconia episcopalis Vell. y Heliconia acuminata A. Rich. (Fig 6) se encontraron a las orillas de los cursos de agua asociados a los relictos selváticos.

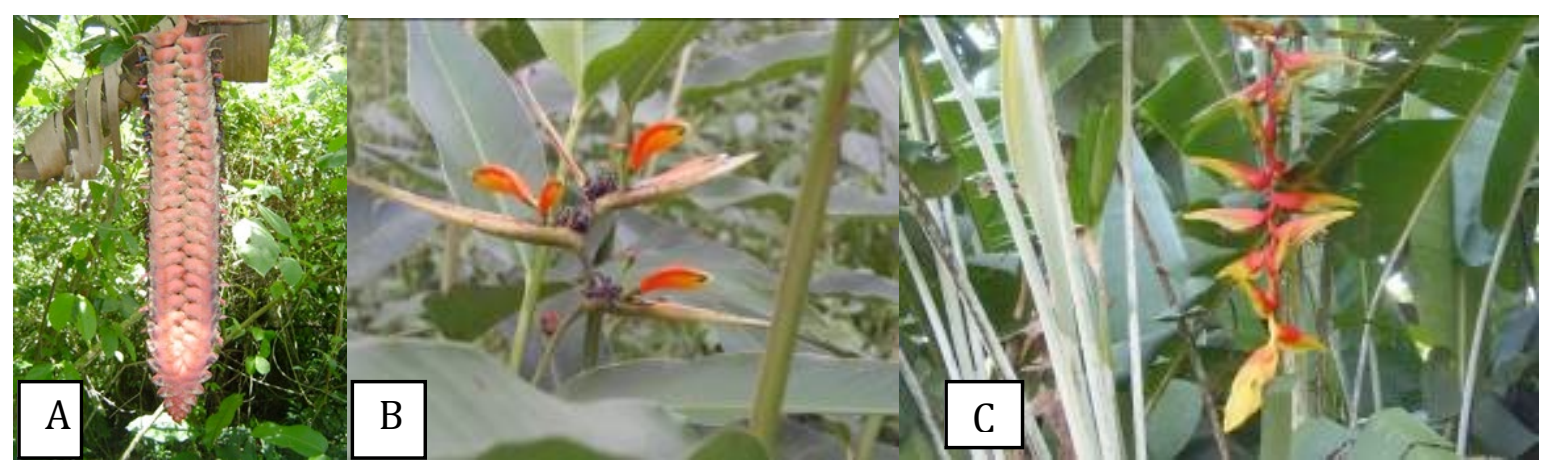

Figura 5. A: Heliconia mariae, B: Helicona hirsuta y C: Heliconia platystachys

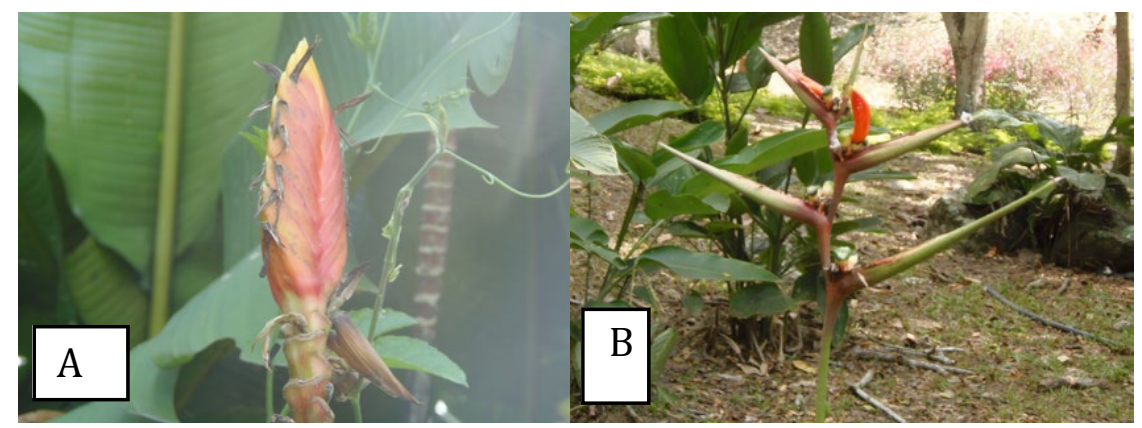

Figura 6. A: Heliconia episcopalis, B: Heliconia acuminata.

De la familia Marantaceae, la especie de mayor distribución es Calathea lutea (Aubl.) E. Mey. Ex Schult. (Fig 7), que se distribuye desde el pie de monte de la Sierra de Mérida hasta las Ciénagas del lago de Maracaibo, mientras que Thalia geniculata L. (Fig 8) sólo se halló al borde de los relictos selváticos que se encontraban ubicados en las planicies aluviales del área de muestreo. Por otra parte Calathea latifolia (Will. ex Link) Klotzsch y Calathea inocephala (Kuntze) T. Durand \& B. D. jacks (Fig 9) se encontraron sólo dentro de los bosques.

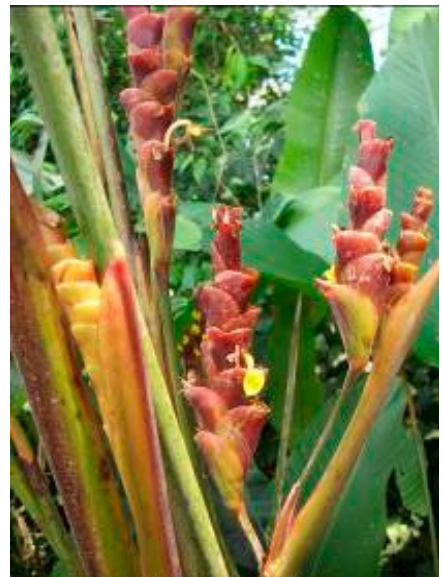

Figura 7. Calathea lutea

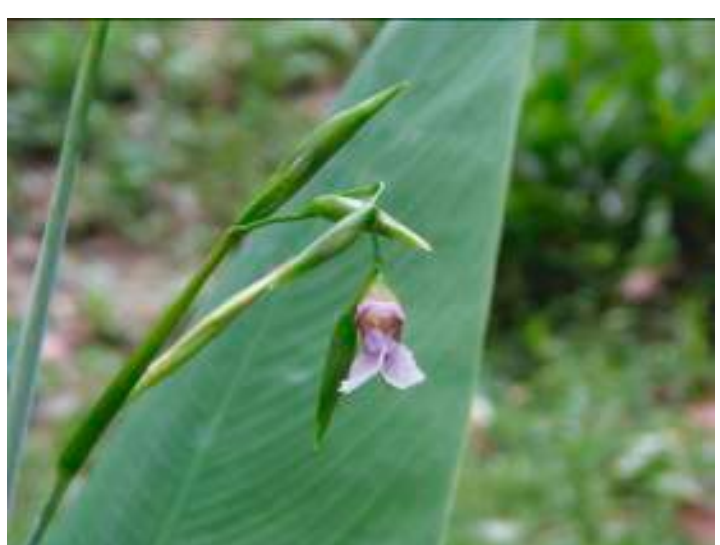

Figura 8. Thalia geniculata 


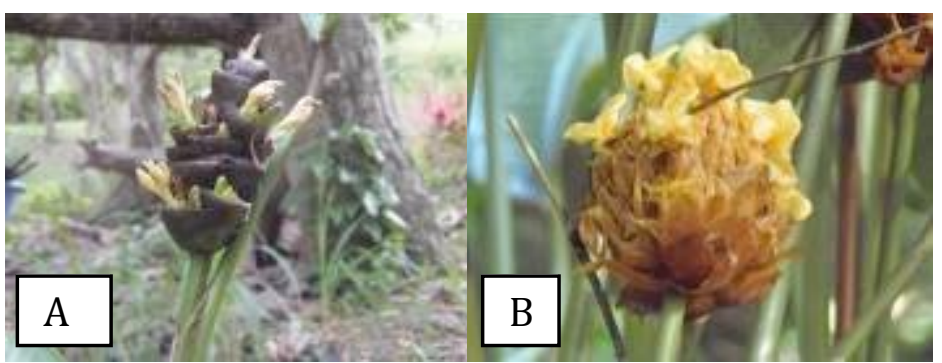

Figura 9. A: Calathea latifolia, B: Calathea inocephala

Para el caso de las Cannaceae, sólo la especie Canna denudata Roscoe (Fig 10) se encontró en los relictos selváticos, al igual que la Zingiberaceae Renealmia thyrsoidea (Ruiz \& Pav.) Poepp. \& Endl. (Fig 11). Esta última especie se registró solo en el pie de monte.

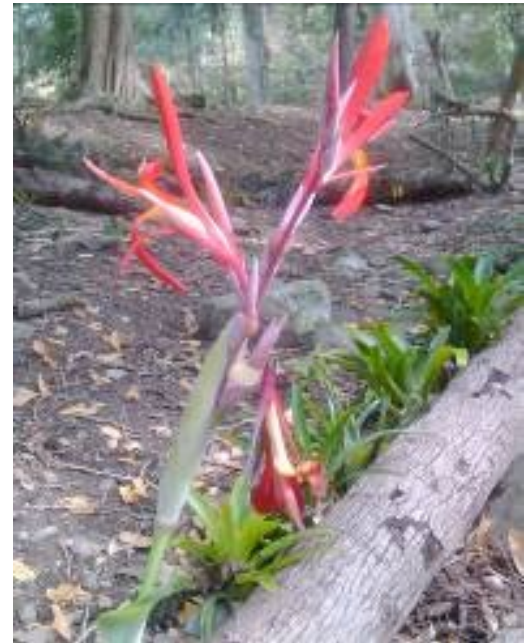

Figura 10. Canna denudata

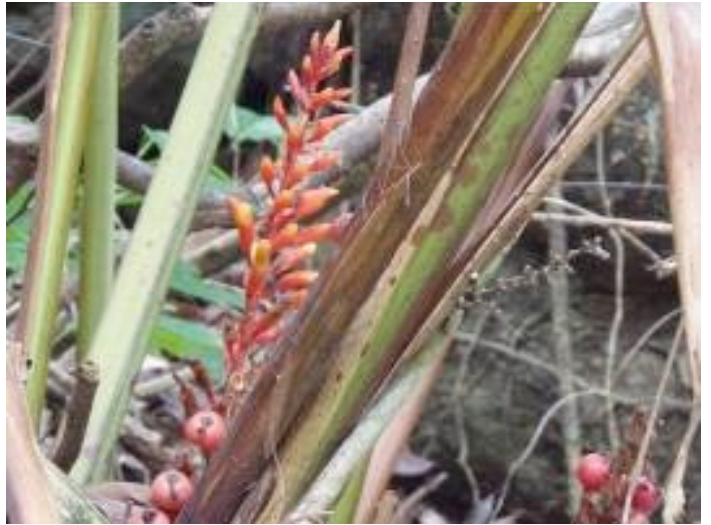

Figura 11. Renealmia thyrsoidea

Las dos especies de Costaceae recolectadas (Fig 12), Costus malortieanus H. Wendl. y Costus spiralis (Jacq.) Roscoe, se encontraron en relictos más cercanos a las laderas de pie de monte que a las Ciénagas del Lago de Maracaibo

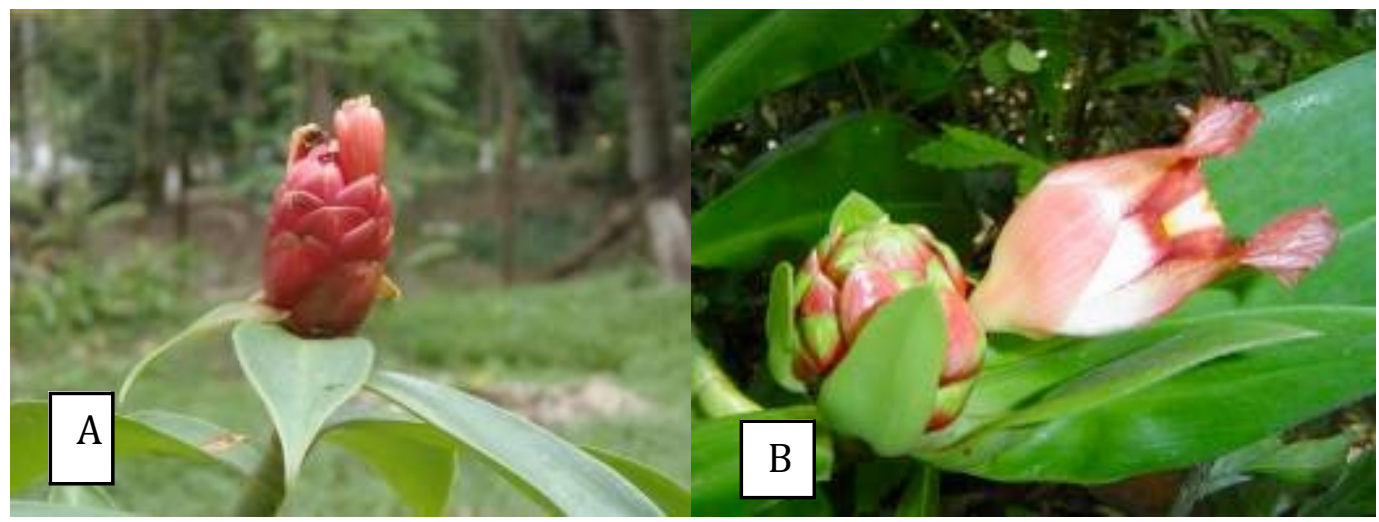

Figura 12. A: Costus malortieanus, B: Costus spiralis 
Todas las especies recolectadas presentan un gran potencial dentro de la floricultura tropical, ya sea como flor de corte, follaje y paisajismo, además de múltiples utilidades ya reportadas, como por ejemplo Calathea lutea es utilizada por los indígenas para extraer fibras y fabricar cestos y un gran número de objetos de artesanía (Hoyos, 1999; Costa et al., 2011), Trujillo y Correa-Muñera (2010) señalan que Renealmia thyrsoidea presenta propiedades medicinales y además a partir de sus frutos se pueden extraer tintes, de igual manera indican que las especies de Costus sp son utilizadas por indígenas de la Amazonía colombiana por sus propiedades curativas.

El valor agregado de este grupo botánico a la flora nativa del Sur del Lago de Maracaibo, podría incentivar a los dueños de las tierras en donde se encuentran los relictos selváticos, a promover su conservación. Algunos autores como Acebey et al. (2007), señalan que el uso de la biodiversidad y de los recursos originados de especies nativas pueden promover la conservación y además traer un beneficio económicos a las comunidades. Un ejemplo de ello es la promoción del cultivo de heliconias nativas de la región central de Cuba en las zonas urbana y periurbanas con la finalidad de contribuir con la conservación amistosa de su biodiversidad Hernández et al. (2015).

\section{REFERENCIAS}

Acebey, A., Kessler, M. \& Maass, B. 2007. Potencial de aprovechamiento de Araceae y Bromeliaceae como recursos no maderables en el bosque montano húmedo del Parque Nacional Cotapata, Bolivia. Ecología en Bolivia 42(1): 4-22.
Costa, F., Penna, F. \& Figueiredo, F. 2011. Guide to the Zingiberales of PPBio sites in Brazilian Western Amazonia. Áttema Design Editorial. 284pg.

De Viana, M., Morandini, M., Giamminola, E. \& Díaz, C. 2011. Conservación Ex situ: un banco de germoplasma de especies nativas. Biodiversidad 1(1): 35-41.

Hernandez, R., Sosa, F., Noa, J., Flores, N., Guillen, D. \& Panfet, C. 2015. Agricultura urbana y periurbana como contribución a la estrategia de conservación de la biodiversidad de Heliconias en la región central de Cuba. Cuadernos de Biodiversidad 47:1-9.

Hoyos, J. 1999. Plantas tropicales ornamentales de tallo herbáceo. Sociedad de Ciencias Naturales la Salle. 592 pp.

Iriondo, J. M. 2001. Conservación de germoplasma de especies raras y amenazadas (Revisión). Invest. Agr.:Prod. Prot. Veg.16(1): 5-24

Kress, W. 1990. The phylogeny and classification of the Zingiberales. Annals of the Missouri Botanical Garden. 77:698-721.

Kress, W. 1985. Bat pollination of an Old World Heliconia. Biotropica. 17:302-308.

Kress, W. 1997. A synopsis of the genus Heliconia (HELICONIACEAE) in Veneuela, with one new variety. Biollania. 6:407-430.

Rangel, S., García-Núñez, C. \& Jaimez, R. 2014. Ecofisiologia de Heliconia bihai L. en el Sur del Lago de Maracaibo. Editorial Académica Española. 101pp.

Romero, L. 1995. El ecosistema selvático del Sur del Lago de Maracaibo y sus sistemas de reemplazo: balance de una transformación. Tesis de maestría. Universidad de los Andes. 148 pp.

Trujillo-C., W. \& Correa-Muñera, M. 2010. Plantas usadas por una comunidad indígena Coreguaje en la Amazonía Colombiana. Caldasia. 32(1): 1-20. 